\title{
Reabilitação estética de diastemas anterossuperiores com resina composta após abordagem ortodôntica
}

\author{
- Leonardo de Almeida Universidade Estadual do Oeste do Paraná (Unioeste), Cascavel, Brasil • Bianca Medeiros \\ Maran Universidade Estadual do Oeste do Paraná (Unioeste), Cascavel, Brasil • Guilherme Schmitt de Andrade Universidade \\ Estadual Paulista "Julio de Mesquita Filho" (Unesp), São José dos Campos, Brasil • Fabiana Scarparo Naufel Universidade \\ Estadual do Oeste do Paraná (Unioeste), Cascavel, Brasil • Vera Lúcia Schmitt Universidade Estadual do Oeste do Paraná \\ (Unioeste), Cascavel, Brasil
}

RESUMO | O sorriso, além de ser um importante fator na autoestima do ser humano, atualmente tem sido considerado a "porta de entrada" para muitas oportunidades. Nesse contexto, existem algumas alterações que podem gerar o comprometimento estético, funcional e psicológico do indivíduo, sendo uma delas o diastema, definido pela presença de espaçamento entre os dentes adjacentes. Objetivos: mostrar, por meio de um caso clínico, uma reabilitação estética direta em paciente jovem com presença de diastemas nos dentes anterossuperiores. Materiais e métodos: realizou-se a reanatomização com resina composta Empress Direct de inserção direta, empregando o auxílio de guia palatina feita em silicone, ocasionando o mínimo de desgaste da estrutura dental. Resultados: o resultado foi satisfatório, tanto para o paciente quanto para o profissional, comprovando a eficiência das resinas compostas em casos de reabilitação, quando bem indicadas. Conclusão: com o aumento na qualidade das técnicas e dos materiais estéticos diretos, seu uso subsequente ao tratamento ortodôntico - caso os pacientes ainda apresentem espaçamentos entre os dentes - proporciona facilidade e segurança ao profissional na realização do procedimento, obtendo ótimos resultados.

DESCRITORES | Diastema; Estética Dental; Resina Composta.

ABSTRACT | Aesthetic rehabilitation of anterosuperior diastemas with composite resin after orthodontic approach • Smile, besides being an important factor in the self-esteem of human, has been considered the "ateway" to many opportunities today. However, there are some changes can be found and which can provide the aesthetic, functional and psychological impairment of the individual, like the diastema, defined by a spacing between adjacent teeth. Objectives: thus, we intend to show through a case an aesthetic rehabilitation of a young patient with the presence of diastema anterosuperiores teeth using composite resin of direct insertion and minimal wear of dental structure. Results: the result was extremely satisfying for both, the patient and the professional, proving the efficiency of composite resins in cases of rehabilitation when well indicated. Conclusion: thus, with the increase in the quality of technical and aesthetic materials, the use of this, subsequent to orthodontic treatment in patients who still show gaps between the teeth, provides ease and security to the professional in performing the procedure, obtaining better results.

DESCRIPTORS | Diastema; Dental Aesthetics; Composite Resin.

AUTOR CORRESPONDENTE | • Leonardo de Almeida Universidade Estadual do Oeste do Paraná • Rua Universitária, 2069 Cascavel, PR, Brasil • 85814-110 E-mail: leonardoalmeida.tem@gmail.com

\footnotetext{
- Recebido $14 \mathrm{jul} .2019$ • Aceito 13 nov. 2019

- Dol http://dx.doi.org/10.11606/issn.2357-8041.clrd.2019.159943
} 


\section{INTRODUÇÃO}

O tratamento dentário pode ser definido pela tríade: saúde, função e estética (SFE), ou seja, o objetivo principal de qualquer tratamento é a resolução da doença, resultando em um estado de bem-estar. ${ }^{1}$

O objeto da estética, segundo Hegel, é o belo artístico criado pelo homem, e ela sempre foi fator fundamental para o ser humano. Porém a estética dental sempre ficou de lado, principalmente quando comparada com o fator "dor". 1,2,3

Com os avanços da odontologia e a diminuição da busca pelo alívio da dor, a estética dental vem se tornando o principal motivo pelo qual o paciente busca um consultório odontológico. ${ }^{4}$

Atualmente, o paciente já procura um cirurgiãodentista com suas vontades e desejos, com uma autoimagem formulada, muitas vezes manipulada pela sociedade, mídia e outros meios. ${ }^{1}$ Essa busca pode ser causada por diversos desequilíbrios, como a assimetria e desproporcionalidade dos dentes entre si e com a gengiva, os lábios e o rosto, estando diretamente associados a alterações relacionadas com posição, forma, tamanho, cor e texturização dos elementos dentais. 5

No agrupamento das alterações de forma, os diastemas dentais são definidos como uma ausência de contato entre dentes adjacentes. Estes podem ser observados em qualquer região da boca, contudo ocorre maior comprometimento estético a partir do momento em que se encontram na região anterior da maxila. ${ }^{6}$

Os tratamentos ortodônticos, de forma geral, promovem uma melhora estética na composição dento-facial. No entanto, nos casos com Discrepância de Bolton, espaços entre os dentes anteriores são ocorrência comum mesmo após o tratamento ortodôntico. Nessas situações, uma avaliação estética para intervenção ortodôntico-restauradora é necessária para distribuir os espaços e permitir restaurações proporcionais e harmônicas.7
Com o passar dos anos, a evolução da qualidade dos materiais restauradores como a resina composta se tornou claramente visível, podendo ser observado o aumento na facilidade de manipulação e maior estabilidade de cor. Com isso, o tratamento para correções de tamanho e forma dos dentes anteriores visando o fechamento de diastemas pode ser realizado de forma minimamente invasiva, preservando a estrutura dental por meio da técnica direta, utilizando sistema adesivo e resina composta. ${ }^{8,9,10}$

\section{RELATO DE CASO}

Paciente do gênero feminino de 15 anos de idade, tendo concluído tratamento ortodôntica há cerca de um mês, procurou atendimento odontológico com sua principal queixa: a insatisfação com seu sorriso devido ao formato de seus dentes.

No exame clínico inicial, observaram-se os incisivos laterais superiores (12 e 22) e centrais superiores (11 e 21) pequenos e com pouco volume, ocasionando diastemas na região anterossuperior de canino a canino (Figura 1), resultando em desarmonia no sorriso, percebendo-se, assim, que a paciente necessitaria de uma reanatomização dessa região. Realizaram-se radiografias periapicais, pois ela apresentava fratura no dente incisivo central superior (11) com colagem de fragmento. Moldaramse as arcadas superior e inferior da paciente para obtenção do modelo de estudo.

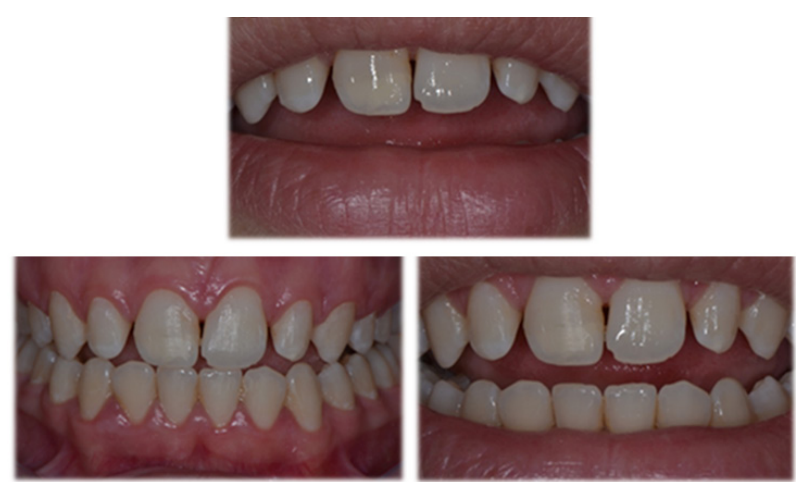

Figura 1 | Aspecto clínico inicial. 
A avaliação radiográfica não indicou nenhuma alteração significante e, considerando ser a paciente ainda muito jovem, optou-se pela reabilitação com resina composta, já que esta oferece ótimos resultados, menor tempo de trabalho e mínimo desgaste da estrutura dental ${ }^{11}$

Realizou-se o enceramento diagnóstico do modelo de estudo, definindo, então, as novas formas e contornos dos dentes (Figura 2), para obter um resultado mais harmônico possível.

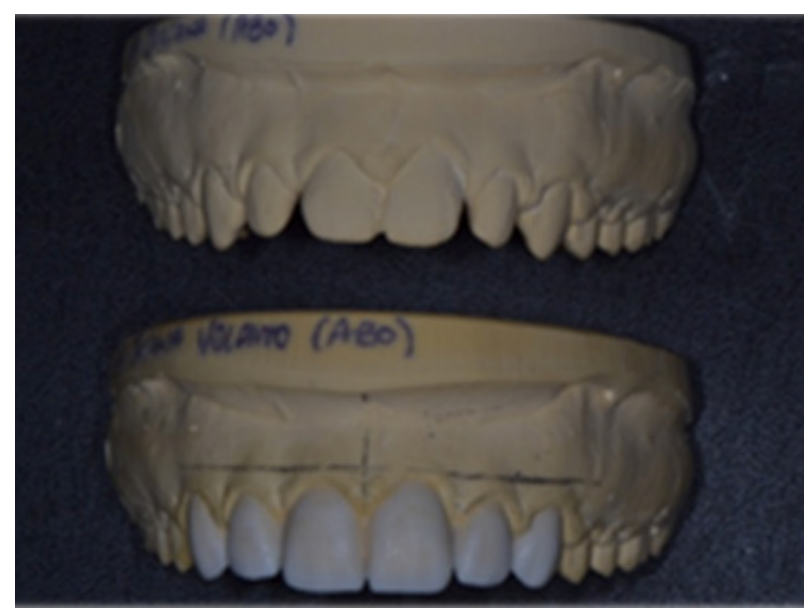

Figura 2 | Enceramento diagnóstico com definição da forma de contorno.

Confeccionou-se sobre o modelo de gesso com enceramento uma guia de silicone com a qual se fez mock-up (Figura 3), usando a resina bisacrílica Protemp 4 (3M ESPE, St. Paul, Minnesota, Estados Unidos), para que tanto o profissional como a paciente observassem o possível resultado a ser conseguido (Figura 4).

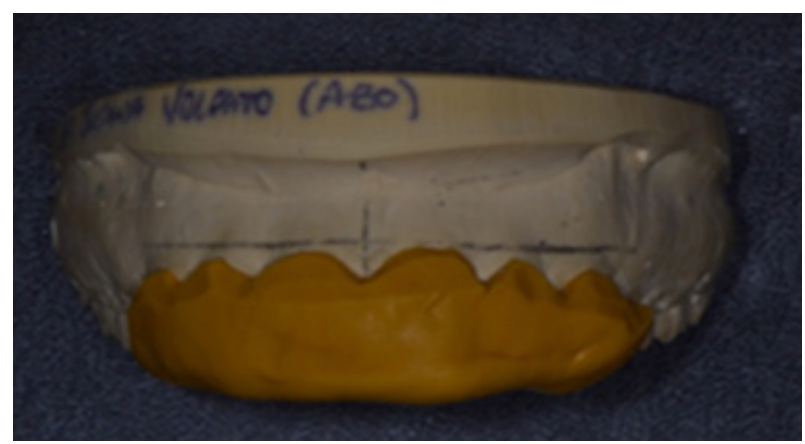

Figura 3 | Confecção da guia de silicone para o mock-up.

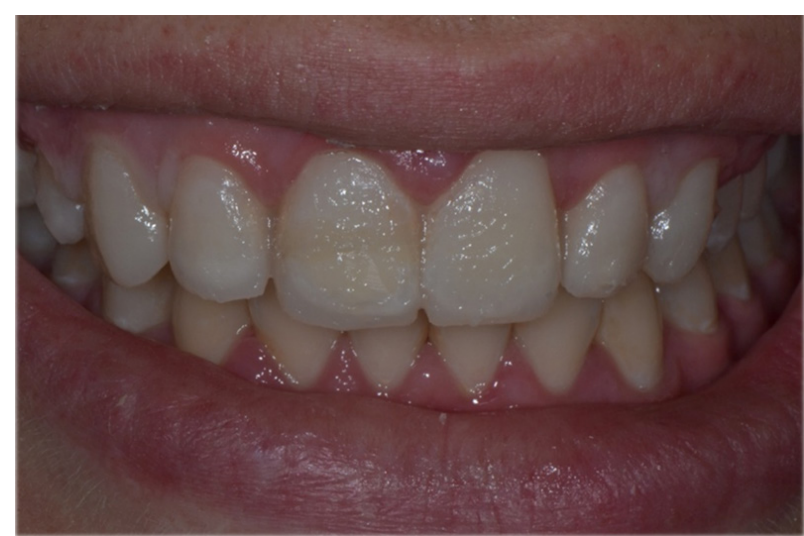

Figura 4 || Aspecto com as novas formas de contorno após o ensaio restaurador.

A paciente usava contenção ortodôntica desde a remoção do aparelho fixo, entretanto, após algum tempo de uso, houve uma pequena desadaptação. Então, para evitar o fechamento fisiológico dos diastemas, optou-se pela confecção de restaurações provisórias com resina composta que serviriam de stops, para manter os espaçamentos até o dia marcado para a realização das facetas definitivas. Os stops foram confeccionados nas mesiais dos dentes 13, 12, 22 e 23 (canino superior direito, incisivo lateral superior direito, incisivo lateral superior esquerdo, canino superior esquerdo) (Figura 5).

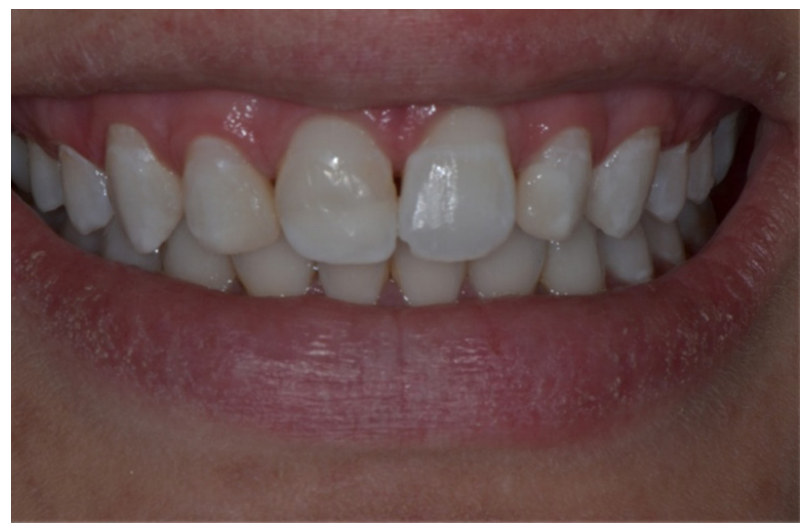

Figura 5 || Aspecto após restaurações nas mesiais dos dentes 13, 12, 22 e 23 (canino superior direito, incisivo lateral superior direito, incisivo lateral superior esquerdo, canino superior esquerdo).

Na seguinte sessão, os stops foram removidos. Em seguida, realizou-se a seleção da resina composta a ser utilizada. 
Optou-se, então, pela resina composta Empress Direct (Ivoclar Vivadent, Schaan, Liechtenstein). Depois, selecionaram-se as cores, pela técnica de "bolinhas", elegidas em cima do dente natural (Figura 6), umedecido e com luz ambiente, sendo, então, escolhidas as cores $\mathrm{DB}_{1}, \mathrm{DA}_{1}, \mathrm{~EB}_{1}, \mathrm{EA}_{1}$ e BL-L.

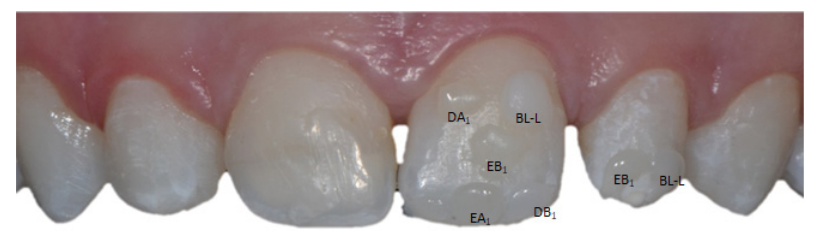

Figura 6 | Seleção de cores.

Após a anestesia e o isolamento absoluto, realizou-se leve desgaste na linha de transição do elemento 11 (incisivo central direito) e asperizações dos outros elementos (Figura 7).

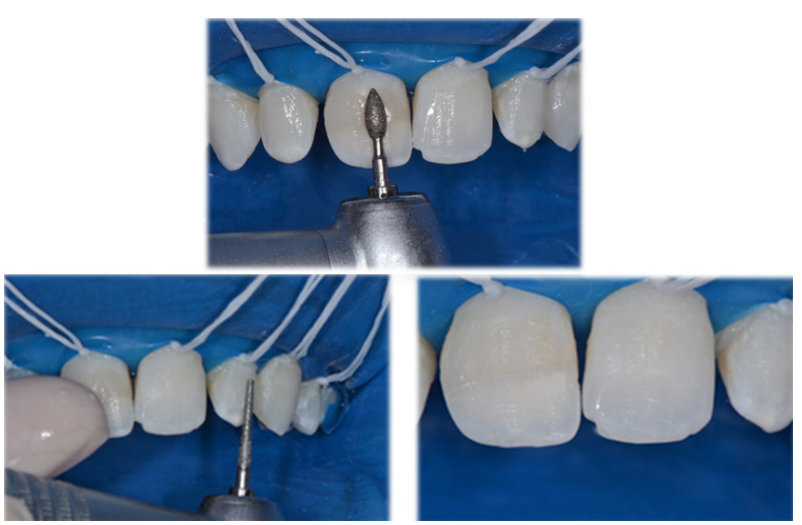

Figura 7 | Desgaste na linha de transição do elemento 11 (incisivo central direito) e asperizações nos dentes a serem trabalhos.

Iniciou-se o condicionamento dos elementos dentais com ácido fosfórico a 37\%, durante 30 segundos, em todo o esmalte vestibular, e porção incisal do esmalte palatino (Figura 8). Em seguida, realizou-se a lavagem abundante com água de todos os dentes condicionados por um minuto (Figura 9). Depois, com os dentes devidamente secos após receberem leves jatos de ar e papel absorvente, foi aplicado e friccionado, com o auxílio de microbrush, o primer Adper Scotchbond MultiPurpose (3M ESPE, St. Paul, Minnesota, Estados
Unidos), seguido da evaporação do seu solvente também com leve jato de ar. Em seguida, aplicou-se o sistema adesivo Adper Scotchbond Multi-Purpose (3M ESPE, St. Paul, Minnesota, Estados Unidos), seguido pela fotopolimerização por 20 segundos (Figuras 10 e 11).

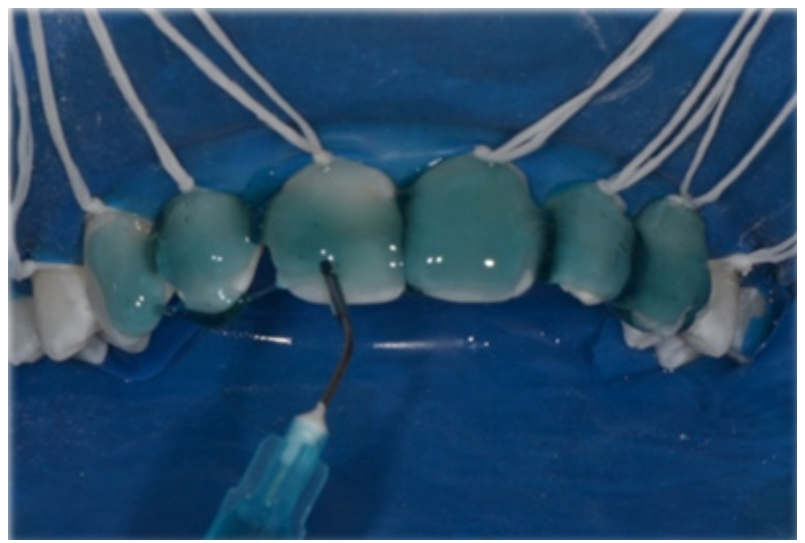

Figura 8 | Condicionamento com ácido fosfórico 37\%.

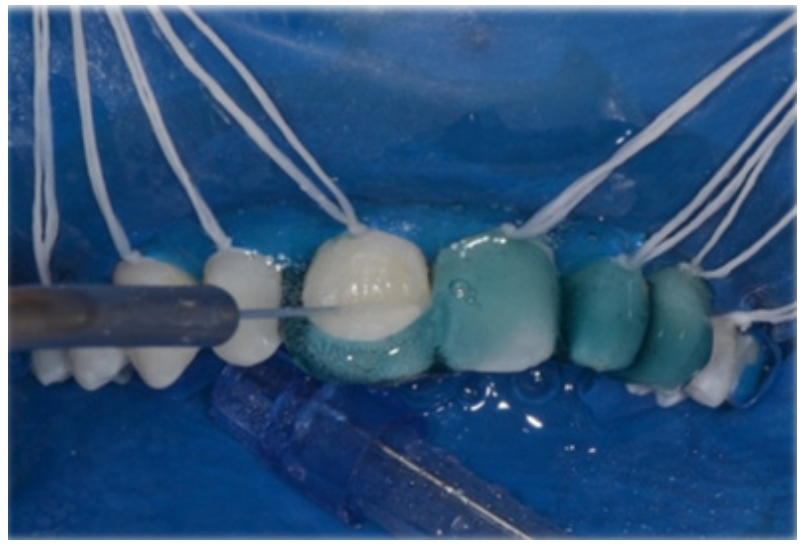

Figura 9 | Lavagem abundante com água.

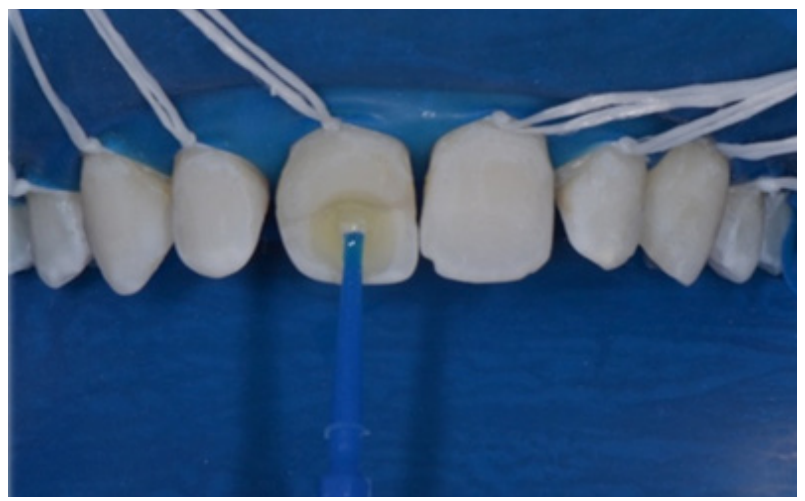

Figura 10 | Aplicação do adesivo. 


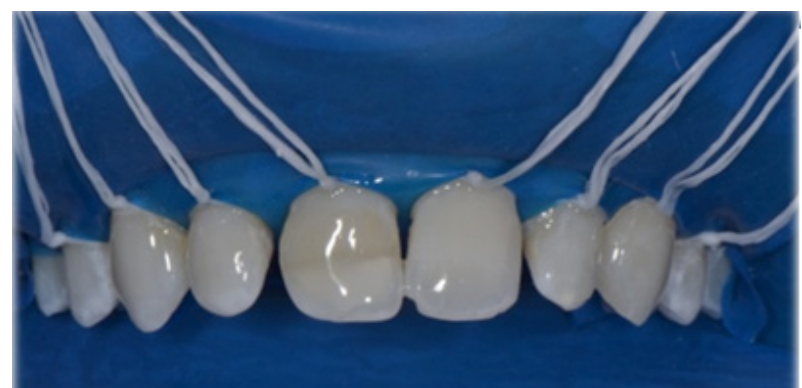

Figura 11 | Aspecto após a aplicação do sistema adesivo.

Após o condicionamento ácido e a distribuição do adesivo, a primeira camada de resina composta começou a ser aplicada, utilizando-se a cor $\mathrm{EB}_{1}$, que foi colocada na guia de silicone com o auxílio de espátula e pincel para então reproduzir o esmalte palatino (Figuras 12) e começar a dar a forma do dente.

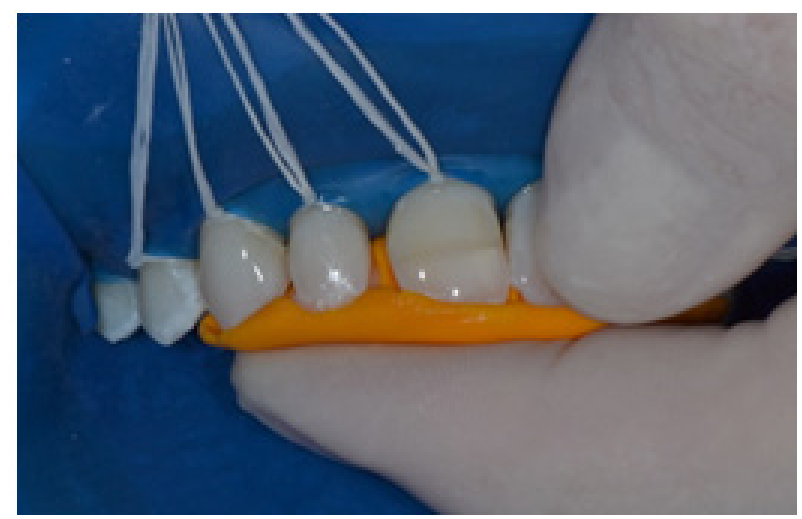

Figura 12a | Guia em posição.

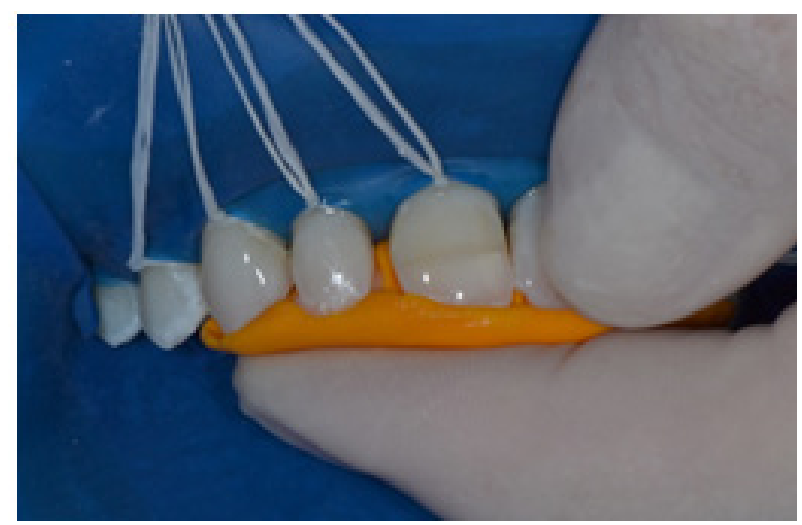

Figura 12b | Primeira camada (palatina) sendo realizada com auxílio da guia de silicone.

Em seguida, inseriu-se a resina composta de cor $\mathrm{DA}_{1}$ na porção cervical e $\mathrm{DB}_{1}$ na porção central, com características um pouco mais opacas para criar uma extensão natural de dentina. Utilizaram-se as resinas $\mathrm{EA}_{1}$ e $\mathrm{EB}_{1}$ para reproduzir a camada de esmalte vestibular e proximais, também com o auxílio de pincel, além de matriz de poliéster.

Por fim, a última camada de resina composta a ser utilizada nesse procedimento de reanatomização foi da cor BL-L, para um melhor resultado estético da reanatomização direta (Figura 13).

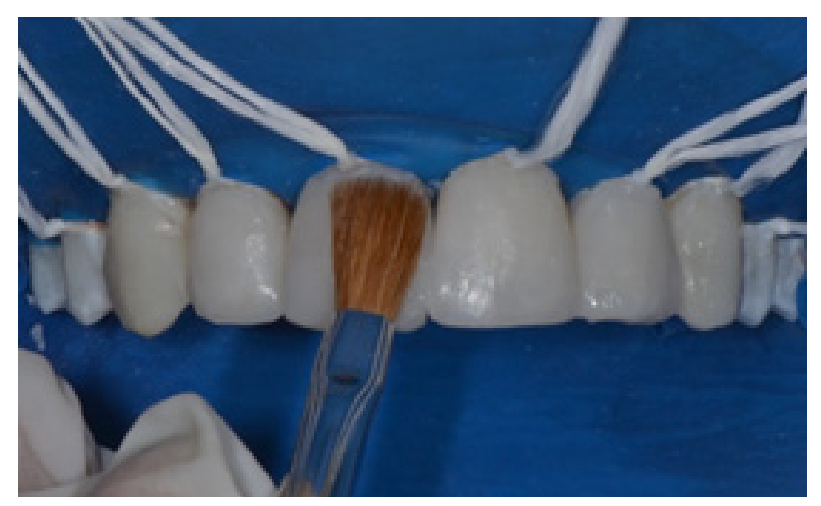

Figura 13 | Término da reanatomização.

Com o término da reanatomização, removeu-se o isolamento e, com o auxílio de lâmina de bisturi número 12, lixa de acabamento, polimento e discos de polimento Sof-Lex (3M ESPE, St. Paul, Minnesota, Estados Unidos), foi realizado acabamento prévio para remoção de excessos. Feito isso, foi checada a oclusão com papel carbono em máxima intercuspidação habitual (MIH), lateralidade e protrusão (Figura 14).

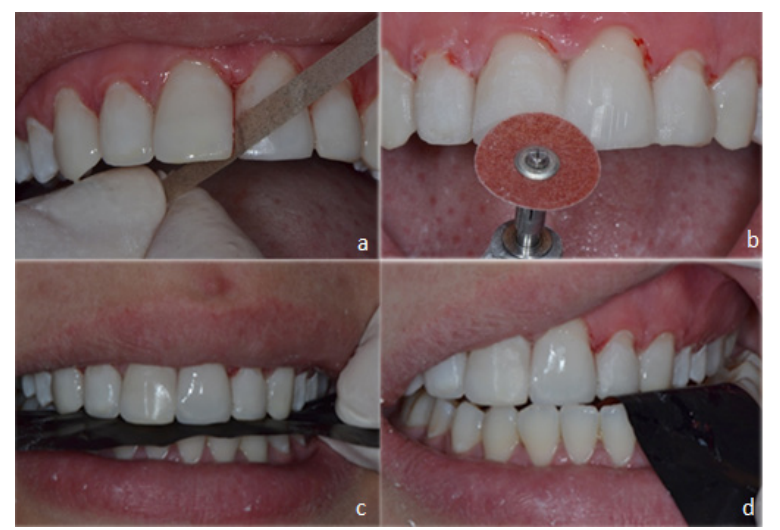

Figura 14a || a) Utilização de lixa de acabamento; b) utilização de discos de lixa; c) checagem de oclusão em protrusão; d): checagem de oclusão em lateralidade. 
Após 21 dias a paciente retornou e, com o uso de uma broca diamantada, foram confeccionadas ranhuras na superfície vestibular dos dentes incisivos superiores para permitir a reflexão da luz, dando uma condição natural à superfície dental. Em seguida, para o acabamento e o polimento final, utilizaram-se discos de lixa Sof-Lex (3M ESPE, St. Paul, Minnesota, Estados Unidos) e discos de feltro Diamond, concluindo a reabilitação com resultado estético satisfatório (Figura 15).

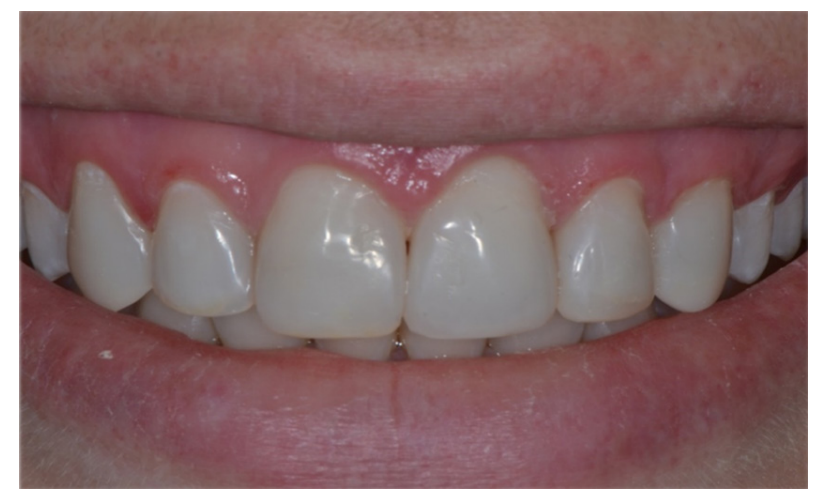

Figura 15 | Aspecto final da reanatomização após acabamento final.

\section{DISCUSSÃO}

As resinas compostas constituem um excelente material restaurador para reabilitações orais estéticas, pois o tempo de trabalho é reduzido, o reparo é fácil, quando necessário, e o desgaste da estrutura dental é mínimo ou nulo, além da mimetização da cor da estrutura dental por parte de sua ampla escala de cores, mesmo quando comparada com outros materiais como as cerâmicas odontológicas, que também oferecem uma excelente mimetização, porém com exigência de desgaste de superfície dental, inviabilizando, em alguns casos, seu uso em pacientes jovens. ${ }^{10-12,16}$

Considerando-se, portanto, a qualidade alta das resinas encontradas hoje no mercado, as quais apresentam boa estabilidade de cor, resistência ao desgaste superficial, cores diversificadas e opacidade adequada, ocorre o favorecimento, desta maneira, na busca de um excelente resultado estético e com ótima durabilidade. Entretanto a qualidade em longo prazo desse material tem a limitação de ser totalmente dependente do paciente, pois, se este não tiver uma boa higiene oral, favorecerá a degradação da matriz orgânica da resina e a consequente alteração da sua cor e textura. ${ }^{5}$

Entretanto é importante salientar que o bom resultado vai depender tanto da colaboração do paciente quanto do conhecimento das técnicas e materiais utilizados pelo profissional, que deve sempre procurar meios que o auxiliem na busca do melhor resultado estético. ${ }^{13}$

Vale ressaltar também que o enceramento diagnóstico serve como uma previsão do resultado final e para a elaboração da guia de silicone, facilitando a reconstrução da face palatina. Como parte do planejamento reverso, o ensaio restaurador intraoral, ou mock-up, é um mecanismo de simulação do planejamento inicialmente proposto, no qual o cirurgião-dentista construirá provisoriamente novas faces aos elementos dentários que necessitam de reabilitação, obtendo, assim, maior previsibilidade do resultado final. Auxilia no planejamento funcional e estético, por meio da avaliação dos parâmetros oclusais necessários a cada caso, além de permitir que o paciente adeque suas expectativas ao planejamento proposto, com maior controle das espessuras de resina e transferência da dimensão mésio-distal e cérvicoincisal para o dente a ser restaurado, auxiliando no procedimento restaurador. ${ }^{14-16}$

\section{CONCLUSÕES}

Abordagem multidisciplinar e planejamento detalhado são sempre muito importantes para se conseguir um resultado estético bom e duradouro. A técnica restauradora direta utilizando resinas compostas, quando corretamente indicada, é uma alternativa de tratamento satisfatória, tendo como principais vantagens a preservação da estrutura dental e o baixo custo. 


\section{REFERÊNCIAS}

1. Ahmad I. Protocolos para restaurações estéticas previsíveis. Porto Alegre: Artmed; 2009.

2. Barbosa R. A filosofia clássica alemã e a crítica estética da modernidade de Schiller a Hegel. Rev Etic Filosof Pol. 2018;1(21):78-93.

3. Resende A. Notas sobre o conceito de "fantasia" nas preleções sobre a estética de Hegel. Artefilosofia. 2017;(4):75-9.

4. Higileh YA. A estética do sorriso [dissertação de mestrado]. Porto: Universidade Fernando Pessoa; 2017.

5. Bernardes ACTAA. Seleção de cor para a obtenção de restaurações anteriores diretas imperceptíveis. Gandra: Instituto Universitário de Ciências da Saúde; 2017.

6. Prado EMA, Melo JCMRD, Soares GP. Fechamento de diastema e reanatomização de dente conoide: relato de caso clínico. Aracaju: Centro Universitário Tiradentes; 2017.

7. Pizzol KEDC, Gonçalves JR, Santos-Pinto A, Peixoto AP. Análise de Bolton: uma proposta alternativa para a simplificação de seu uso. Dental Press J Orthod. 2011;16(6):69-77. doi: 10.1590/S2176-94512011000600012.

8. Diegues MA, Marques E, Miyamotto PAR, Penteado MM. Cerâmica x resina composta: o que utilizar? Uningá Rev. 2018;51(1):87-94.

9. Da Silva GR, Fracalossi C, De Lucena MTL, Mantovani MB. Tratamento estético com diretas de resina composta: relato de caso. Uningá Rev. 2018;24(3):27-31.
10. Leite ADS. Facetas diretas com resina composta em dentes anteriores superiores: estudo de caso [trabalho de conclusão de curso]. Santa Cruz do Sul: Universidade de Santa Cruz do Sul; 2017.

11. Cassiano A, Canani JK, Amaral RC, Cecconello R, Comunello SMH, Oliveira Ramos G, et al. Estratificação em resina composta para reanatomização de dente anterior escurecido: relato de caso. Ação Odonto. 2017;(2):30-37.

12. Marin F, Honorato JR. Faceta de porcelana: indicações e contraindicações. Porto Velho: Centro Universitário São Lucas; 2017.

13. Moura ABD. Facetas de resina composta ou cerâmicas: qual utilizar? [trabalho de conclusão de curso]. Florianópolis: Universidade Federal de Santa Catarina; 2017.

14. Pereira TM, Bezerra RB, Machado AW. Esthetic rehabilitation of anterior teeth with porcelain laminates: case report. J Dent Pub Health. 2018;9(3):195-204. doi: 10.17267/2596-3368dentistry.v9i3.1742.

15. Mathias AP, Tsuzuki FM, Souza BAV, Souza Biguetti G, Carvalho JCZ, Sábio SS, et al. Reabilitação estético-funcional com e-max. Uningá Rev. 2018;55(1):155-65.

16. Reis GR, Oliveira LPM, Vilela ALR, Menezes MS. Mockup: previsibilidade e facilitador das restaurações estéticas em resina composta. Rev Odontol Bras Central. 2018;27(81):105-11. 